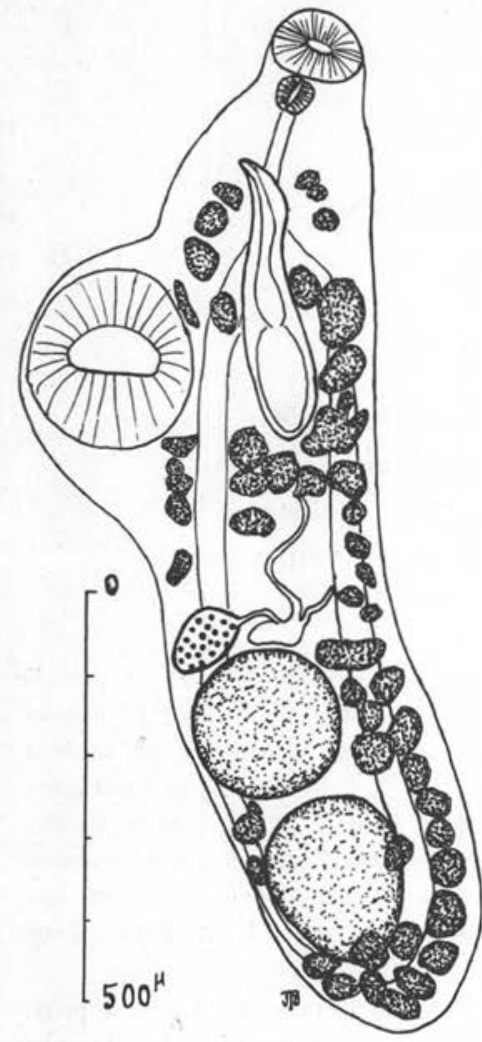

Métacercaire de Nicolla gallica $\mathrm{R}$.-Ph. Dollfus, tirée de son kyste chez Gammarus pulex L. Marais entre Berre et Saint-Chamas (B.-du-Rh.)
Le Mollusque Theodoxia fluviatilis n'existe pas dans le gîte qui a fourni les Gammares, mais on y trouve en abondance Pseudamnicola compacta (Pal.). Ces Prosobranches renferment des sporocystes blanchâtres, longs de 1 à $2 \mathrm{~mm}$, où se développent des cercaires cotylicerques identiques à celles qui ont été trouvées à Richelieu chez Theodoxia : vessie ovoïde avec épaisse paroi épithéliale, quatre paires de glandes de pénétration, court stylet (15 $\mu$ et long prépharynx, acétabulum protractile.

Cette nouvelle station de Nicolla gallica dans le département des Bouches-du-Rhône s'ajoute à celle qui a été signalée par P. Mathias (19361937) aux environs de Montpellier. L'espèce doit être assez répandue dans le Midi de la France.

Jean TrMon-DaviD.

\title{
Problèmes de Nomenclature : Armillifer L. W. Sambon, 1922 ou Nettorhynchus (H. D. de Blainville, 1824) J. C. Zenker, 1827 emend.?
}

Parmi les quatorze genres de Porocephaloïdea, le genre Armillifer L. W. Sambon, 1922 est, à la fois, le plus connu et le plus répandu. Deux des trois espèces collectives le composant sont décrites depuis longtemps: A. moniliformis par C. M. Diesing en 1835, A. armillatus par J. Wyman en 1845 (1). Seule l'espèce $A$. grandis est de connaissance plus récente puisqu'individualisée en 1915 seulement par Miss M. L. Hett.

(1) Et non 1847 comme il est écrit souvent par erreur. En effet, le titre original est celui-ci « Notice of two species of Linguatula. By Jeffries Wyman, M.D. Communicated September 17, $1845 »$, in Boston Journal of Natural History, tome V, 294-296. 
Or, en 1950, R.-Ph. Dollfus crut possible de reprendre un nom méconnu dû à H.-D. de Blainville, 1824 (Nettorhynque) pour désigner le genre Armillifer de L. W. Sambon. Sur cette assurance, quelques auteurs (dont l'un de nous) utilisèrent le genre Nettorhynchus et même la famille des Nettorhynchidae pour Armilliferidae fut créée par J. Doucet en 1962.

Cependant, préparant une notice pour le centenaire de la mort du naturaliste Alfred Moquin-Tandon (2), nous devrions trouver dans les Eléments de Zoologie médicale de cet auteur (Paris, 1860, pages 380-381) quelques phrases qui ne nous permettaient plus d'accepter sans réticence la synonymie nouvelle.

Après bien des recherches, il nous fut possible de réunir la totalité des documents relatifs au genre Nettorhynque. Nous allons très brièvement les résumer.

Le genre Nettorhynque a été défini par H.-D. de Blainville en 1824 dans l'Appendice au Traité zoologique et physiologique sur les vers intestinaux de l'Homme de M. Bremser, traduction Grundler, page 522, pour un «ver extraordinaire » décrit et figuré par John Paisley*, chirurgien à Glasgow vers 1734 .

Le texte de J. Paisley, non disponible en France, nous a été communiqué obligeamment par les services de l'Ecole de Médecine de l'Université d'Edinburgh. Il y est question d'un «ver» émis mort par un jeune duelliste ayant reçu une blessure quelques semaines auparavant, blessure infectée et suivie d'ictère et d'un second «ver» désagrégé émis par la suite en morceaux. Les dimensions indiquées sont notables ( 1 pied 6 pouces de long sur 1 pouce $1 / 2$ de diamètre). Rien ne permet de retrouver dans cet objet un Armillifer parasite des gros serpents d'Afrique ou d'Asie. D'autant que, comme le dit J. Paisley, la figure publiée n'est pas absolument conforme à la réalité : «The Interstices between each Joint were rather larger than as they appear in the Figure and were of a dark Chocolate Colour; t the Joints themselves more pale, or rather of a livid Flesh-colour... »

Le texte de H.-D. de Blainville qui prétend reproduire les paroles de J. Paisley est un peu « amélioré ». Les commémoratifs de l'événement (duel à l'épée) ne sont plus indiqués. Quelques détails morphologiques sont ajoutés et la dimension du « ver » est exagérée (2 pieds 6 pouces).

Au-delà, les données de H.-D. de Blainville seront reprises et discutées par divers suteurs sans qu'il soit question du texte original du vieux chirurgien écossais (J.-C. Zenker, ז827, Ch. Le Blond, 1837, F.-V. Raspail, 1846, P. Gervais, 1846, Dubini, 1849, Alfr. MoquinTandon, 1860, Th.-Sp. Cobbold, 1864).

Dans ces conditions, il nous paraît impossible de maintenir le genre Nettorhynchus, celui-ci se rapportant à un élément certainement différent du genre Armillifer, élément sur la nature duquel nous nous réservons d'intervenir plus longuement ultérieurement, car il sou-

(2) Notice présentée lors de la première session de la Société française de Parasitologie. Rennes, 1963.

* Note. - Le nom même de John Paisley est pratiquement inconnu. Issu de l'Université de Glasgow, membre de la Faculty of Physicians and Surgeons at Glasgow, J. Paisley fut un chirurgien de grand renom. Il fut nommé bibliothecarius de la Faculté et put enseigner officiellement l'anatomie entre 1730 et 1740 . L'un de ses élèves fut le très célèbre William Cullen (1710-1790).

Remerciements: With hearty acknowledgements to the staff of the Central Medical Library of the University of Edinburgh and especially to Miss M. D. Bell, sub-Librarian of the Medical Libraries, without whose co-operation this study would have been incomplete. 
lève un très curieux problème d'Histoire de la Médecine et tout particulièrement de la Parasitologie, problème méconnu à ce jour.

R. M. Nicoli, Ph. Latourelle et M. Quilici.

(Laboratoire de Parasitologie. Faculté mixte de Médecine et de Pharmacie de Marseille)

\section{Bibliographie}

Blainville H.-D. DE, 1824. - Appendice au Traité zoologique et physiologique sur les Vers intestinaux de M. Bremser, traduction Grundler, Paris, 522.

Dollfus R.-Ph., 1950. - Armillifer L.W. Sambon, 1922, tombe en synonymie de Nettorynque H. D. de Blainville, 1824. Ann. Parasit. hum. comp., XXV, 112-113.

Doucet J., 1962. - Pentastomes de l'Angola. Publ. cult. Co. Diam. Ang., 115-120.

DUBINI, 1849. - Entozoografia umana per service di complemento agli studi di anatomia patologica. Milano.

GervaIs P., 1846. - Nettorhynque. Dictionnaire Universel d'Histoire Naturelle. VIII, 630.

LE BLond Ch., 1837. - Traité zoologique et physiologique sur les Vers intestinaux de l'homme. Atlas avec texte explicatif, $2^{2}$ édition, $54-55$, pl. XIV.

MoQuin-Tandon Alfr., 1860. - Eléments de Zoologie médicale. Paris, 380-381.

Nicoli R. M., Golvan Y.-J., 1963. - Pentastomides de l'Angola. Publ. cult. Co. Diam. Ang.

PAISLEY J., 1734. - An Account of an extraordinary Worm. Edinb. Medic. Essays and Observations. II, 333-339, pl. IV.

RASPAIL F. V., 1846. - Histoire naturelle de la santé et de la maladie chez les végétaux et chez les animaux en général, en particulier chez l'homme, $2^{c}$ édition. II, 442-443.

SAmbon L. W., 1922. - A synopsis of the family Linguatulidae. Jl. of Trop. Med. Hyg. XXV, 188206 et $391-428$.

ZENKER J.C., 1827. - Parasitae corporis humani internae seu vermes intestinales hominis. Lipsiae.

\section{Présence en France de Exopalpiger trianguliceps}

\section{(Birula, 1895) (Acariens, Ixodoidea)}

Exopalpiger trianguliceps (Birula, 1895) est connu de la plupart des pays d'Europe jusqu'en Sibérie : Allemagne (Schulze, 1933, 432, Ixodes nivalis ; Schulze, 1930, 1-18, I. nivalis ; Neumann, 1901, 249 : 286, Ixodes tenuirostris ; Schulze, 1939, 35 et 1943, 253, Endopalpiger heroldi), Bulgarie (Černy, 1959, 361 ; Drenski, 1955, 109), GrandeBretagne (Wheler, 1906, 400 ; Neumann, 1901, 249 : 286, Ixodes tenuirostris ; Nuttall et Warburton, 1911: 248, I. tenuirostris ; Nuttall, 1916, 294, I. tenuirostris ; Evans, 1907, 36 ; I. trianguliceps; ; Warburton, 1926, 55, I. tenuirostris ; Hora, 1934, 361, I. tenuirostris ; Milne, 1949, 173, I. tenuirostris), Pologne (Filippova, 1958, 10 ; Lachmajer et Wegner, 1959, 175), Danemark (Arthur, 1956, 26), Norvège (Tambs-Lyche, 1943, 37), Suède (Schulze, 1930, 1-18, I. n. suecicus), Italie (Tonelli-Rondelli, 1928, 85 et 1938, 80, I. nivalis ; Starkoff, 1958: 146), Suisse (Nuttall et Warburton, $1911: 248$ ), Tchécoslovaquie (Mačička, 1955, 284 ; Černy, 1959, 156 et 1961, 574 ; Rosicky, 1953, 120). U.R.S.S., de la Baltique et la Karélie jusqu'au lac Baïkal (Pomerancev, 1950 : 81 ; Filippova, 1958, 10 ; Emelhanova et Pauller, 1958, 197 ; Šluger, 1961, 425 ; MalhyoŠina, 1963, 355 ; Nikitina, 1960, 708). 